\title{
Prevalence and Clinical Characteristics of Psoriatic Arthritis in Japan
}

\author{
Yuri Ohara, Mitsumasa Kishimoto, Naoho Takizawa, Kazuki Yoshida, Masato Okada, \\ Hikaru Eto, Gautam A. Deshpande, Christopher T. Ritchlin, Atsushi Tanaka, Mari Higashiyama, \\ Kazuo Matsui, and Shigeyoshi Tsuji
}

\begin{abstract}
Objective. To investigate the prevalence of psoriatic arthritis (PsA) in Japanese patients with psoriasis. Methods. A multicenter, noninterventional, retrospective cross-sectional study was conducted at 3 tertiary care centers in Japan. PsA was diagnosed by rheumatologists based on clinical findings. Prevalence of PsA, clinical characteristics, comorbidities, and treatment patterns were examined.

Results. PsA was identified in 431 of 3021 patients with psoriasis, with a mean prevalence of $14.3 \%$ (range, 8.8-20.4\%). No large differences between these results and previous reports from Western countries were observed in arthritis distribution, skin disease type, or treatment selection.

Conclusion. The prevalence of PsA in patients with psoriasis in Japan approaches $20 \%$ in some areas, similar to that observed in Western countries, and is higher than previously reported in Asia. Clinical features including age, sex, age at onset, and manifestation patterns were also similar to those reported in the West. (J Rheumatol First Release June 15 2015; doi:10.3899/jrheum.141598)
\end{abstract}

Key Indexing Terms:

PSORIATIC ARTHRITIS PSORIASIS PREVALENCE ASIA JAPAN

Psoriatic arthritis (PsA) is a complex musculoskeletal disorder that is usually preceded by psoriasis. The cardinal manifestations of PsA include psoriasis, peripheral arthritis, spinal lesions, enthesitis, and dactylitis. New bone formation is characteristic on imaging ${ }^{1}$. The prevalence of psoriasis among Asians is reported to be about $0.1 \%{ }^{2,3}$, which is lower than the $2 \%$ reported among Westerners ${ }^{4}$. Among Japanese,

From the Immuno-Rheumatology Center, and the Department of Dermatology, St. Luke's International Hospital, Tokyo; Department of Rheumatology, and the Department of Dermatology, Kameda Medical Center, Chiba, Japan; Department of Epidemiology, Harvard School of Public Health, Boston, Massachusetts; Allergy, Immunology, and Rheumatology Division, University of Rochester Medical Center, Rochester, New York, USA; Department of Dermatology, Nissay Hospital; Department of Orthopedics, National Hospital Organization, Osaka Minami Medical Center, Osaka, Japan.

Y. Ohara, MD*, M. Kishimoto, $M D^{*}$, Immuno-Rheumatology Center, St. Luke's International Hospital; N. Takizawa, MD, Department of Rheumatology, Kameda Medical Center; K. Yoshida, MD, MPH, Department of Epidemiology, Harvard School of Public Health; M. Okada, MD, Immuno-Rheumatology Center, St. Luke's International Hospital; H. Eto, MD, Department of Dermatology, St. Luke's International Hospital; G.A. Deshpande, MD, MA, Immuno-Rheumatology Center, St. Luke's International Hospital; C.T. Ritchlin, MD, Allergy, Immunology, and Rheumatology Division, University of Rochester Medical Center; A. Tanaka, MD, Department of Dermatology, Kameda Medical Center; M. Higashiyama, MD, Department of Dermatology, Nissay Hospital; K. Matsui, MD, Department of Rheumatology, Kameda Medical Center; S. Tsuji, MD, Department of Orthopedics, National Hospital Organization, Osaka Minami Medical Center.

*Y. Ohara and M. Kishimoto contributed equally to this study.

Address correspondence to Dr. M. Kishimoto, Immuno-Rheumatology Center, St. Luke's International Hospital, 9-1 Akashicho, Chuo-ku, Tokyo, Japan.E-mail:kishimotomi@gmail.com

Accepted for publication April 22, 2015. the prevalence of psoriasis is also reported to be $0.1 \%$, but may be increasing because of recent Westernization of lifestyle, and other factors ${ }^{5}$. The reported prevalence of PsA among patients with psoriasis ranges from $6 \%$ to $>42 \%$, 7,8 . Specifically, the prevalence among whites is estimated at $15-30 \%$, whereas many studies indicate the prevalence among Asians to be $\leq 10 \% 9,10$; the previously reported prevalence among Japanese patients with psoriasis is $1 \%{ }^{11}$. While these discrepancies are likely due to differences in study populations, they may also be the result of underdiagnosis, owing to an insufficient awareness of PsA in Asia.

We investigated the prevalence of PsA among Japanese psoriatic patients; surveyed the types of psoriasis, varieties of arthritis, and associated comorbidities; and determined whether the Assessment of Spondyloarthritis International Society (ASAS) classification criteria ${ }^{12}$ and the ClASsification for Psoriatic ARthritis criteria (CASPAR) ${ }^{1}$ were met.

\section{MATERIALS AND METHODS}

This was a multicenter, noninterventional, retrospective cross-sectional study. Data from all patients with psoriasis examined between March 2003 and February 2014 at tertiary care centers in 3 major Japanese areas (Tokyo, Osaka, and Chiba) were included. These tertiary care centers represent the flagship psoriasis hospital in each respective area. In the Japanese healthcare system, most patients with skin conditions are able to (and do) access a dermatologist directly without first seeing a primary care physician. The clinical diagnosis of psoriasis was made by dermatologists, distinguishing between psoriasis vulgaris [International Classification of Diseases, 10th edition (ICD-10)-L400], guttate psoriasis (ICD-10-L404), and pustular psoriasis (ICD-10-L401), including palmoplantar pustulosis (ICD-10-L403). Coding, per ICD-10, was done at the time of consultation. Individuals with psoriasis were identified from hospital databases in each tertiary care center. Rheumatologists familiar with the CASPAR made diagnoses of psoriatic

Personal non-commercial use only. The Journal of Rheumatology Copyright @ 2015 . All rights reserved. 
arthritis (PsA) based on clinical manifestations and individual physician judgment.

Evaluation items. The prevalence of PsA among patients with psoriasis was calculated for each institution as well as for pooled data. Dermatologists distinguished psoriasis patterns as described above. Rheumatologists assessed distribution of arthritis, which was further evaluated per Moll and Wright criteria $^{13}$, classifying it as distal interphalangeal arthritis, asymmetrical oligoarthritis, polyarthritis, spondylitis, and arthritis mutilans. The presence of spondylitis, enthesitis, dactylitis, and nail lesions was evaluated per rheumatologist judgment at participating sites. Other variables assessed were obesity, defined per World Health Organization criteria for Asians as a body mass index $(\mathrm{BMI}) \geq 25$; family history of psoriasis within a second degree of relationship; and comorbidities [hypertension (HTN), hyperlipidemia, hyperuricemia, and liver dysfunction] 3 months before or after diagnosis. The prevalence of these comorbidities was assessed in the context of prevalence in the general population as reported in a survey conducted by the Ministry of Health, Labor, and Welfare of Japan. Blood tests were performed to determine the level of rheumatoid factor, anticyclic citrullinated peptide antibodies, matrix metalloproteinase-3, C-reactive protein, and erythrocyte sedimentation rate. Values higher than local reference levels were defined as positive. When arthritis symptoms were present, painful areas were evaluated by radiograph or magnetic resonance imaging at the discretion of the examining physician. The presence of imaging findings typical of PsA was also confirmed (typical peripheral joint new bone formation with or without erosions; sacroiliac joint bone erosions; syndesmophytes on the sacroiliac joint or spine; bamboo spine). Peripheral joint findings conformed to CASPAR criteria, defined as radiological evidence of juxtaarticular new bone formation, i.e., ill-defined ossification near joint margins (but excluding osteophyte formation) on plain radiographs of hand or foot ${ }^{1}$.

Our study conformed to the principles of the Declaration of Helsinki, and was approved by each hospital's ethics committee or institutional review board.

Statistical analyses. The prevalence of PsA was calculated by dividing the number of patients with PsA by the total number of patients with psoriasis examined as of December 31, 2013. Point estimates and 95\% CI were reported. Descriptive statistics of patient characteristics, such as dermatologic and rheumatologic medications, laboratory data, and imaging findings were reported for the overall cohort as well as for each individual institution. For categorical variables, proportions were described as percentages. For imaging findings and diagnostic criteria, proportions were calculated for patients without missing values. For continuous variables, median and interquartile ranges were reported. Associations between comorbidities (obesity, hyperlipidemia, diabetes, hyperuricemia, HTN, and liver dysfunction) were assessed with Fisher's exact test.

\section{RESULTS}

From all participating sites, a total of 3021 patients with psoriasis were enrolled. Of these, 431 had PsA (pooled prevalence, $14.3 \%$; range, 8.8-20.4\%; Table 1). The prevalence of PsA at each participating hospital was Tokyo, 139/1017 (13.7\%; 95\% CI, 12\%-16\%); Osaka, 204/1000 (20.4\%; 95\% CI, 18\%-23\%); and Chiba, 88/1004 (8.8\%; 95\% CI, $7 \%-11 \%$ ).

Table 1 gives the median age at psoriasis onset and arthritis onset, median latency time to arthritis onset, distribution of patients with PsA, distribution of skin disease types, types of arthritis, types of skin lesions, timing of onset, laboratory data, imaging results, and comorbidities. Comorbid diseases were more common among patients with psoriasis than among the general population. Hyperlipidemia
Table 1. Overall characteristics of 431 patients with psoriatic arthritis. Data are median $[\mathrm{IQR}]$ or $\mathrm{n}(\%)$

\begin{tabular}{|c|c|}
\hline \multicolumn{2}{|l|}{ Characteristics } \\
\hline Age, as of December 31, 2013, yrs & $53.00[43.00,64.00]$ \\
\hline Men & $258(59.9)$ \\
\hline Age at psoriasis onset, yrs & $37.00[25.00,51.00]$ \\
\hline Age at arthritis onset, yrs & $45.00[36.00,57.00]$ \\
\hline \multicolumn{2}{|l|}{ Latency time to onset of arthritis } \\
\hline (from psoriasis onset), yrs & $5.00[0.00,13.00]$ \\
\hline Duration of psoriasis, yrs & $13.00[7.00,22.00]$ \\
\hline Duration of psoriatic arthritis, yrs & $5.00[2.00,10.00]$ \\
\hline History of smoking & $163(37.8)$ \\
\hline Obesity & $112(26.0)$ \\
\hline Family history & $42(9.7)$ \\
\hline \multicolumn{2}{|l|}{ Arthritis type } \\
\hline Distal interphalangeal & $38(8.9)$ \\
\hline Oligoarthritis & $122(28.6)$ \\
\hline Polyarthritis & $258(60.4)$ \\
\hline Mutilans & $2(0.5)$ \\
\hline No peripheral arthritis & $3(0.7)$ \\
\hline \multicolumn{2}{|l|}{ Skin lesion } \\
\hline Psoriasis vulgaris & $385(91.9)$ \\
\hline Guttate psoriasis & $2(0.5)$ \\
\hline Pustular psoriasis & $26(6.2)$ \\
\hline No skin lesions & $6(1.4)$ \\
\hline Nail lesions & $168(39.0)$ \\
\hline Dactylitis & $177(41.1)$ \\
\hline Enthesitis & $187(43.4)$ \\
\hline Axial symptoms & $149(34.6)$ \\
\hline \multicolumn{2}{|l|}{ Timing of onset } \\
\hline Psoriasis first & $312(72.9)$ \\
\hline Simultaneous & $69(16.1)$ \\
\hline Arthritis first & $47(11.0)$ \\
\hline \multicolumn{2}{|l|}{ Imaging findings } \\
\hline \multicolumn{2}{|l|}{ Peripheral arthritis (typical new bone formation } \\
\hline Axial joints & $164 / 401(40.9)$ \\
\hline Syndesmophytes in radiograph & $165 / 341(48.4)$ \\
\hline Sacroiliac lesions in radiograph & $127 / 296(42.9)$ \\
\hline Spinal lesions using MRI & $54 / 122(44.2)$ \\
\hline Sacroiliac lesions using MRI & $52 / 151(34.4)$ \\
\hline \multicolumn{2}{|l|}{ Blood tests } \\
\hline Rheumatoid factor-positive & $49(11.9)$ \\
\hline Anticyclic citrullinated peptide antibodies & $23(6.1)$ \\
\hline Elevated C-reactive protein & $195(45.2)$ \\
\hline Elevated matrix metalloprotease-3 & $108(30.5)$ \\
\hline \multicolumn{2}{|l|}{ Concomitant diseases } \\
\hline Hyperlipidemia & $189(43.9)$ \\
\hline Diabetes & $65(15.1)$ \\
\hline Hyperuricemia & $90(20.9)$ \\
\hline Hypertension & $100(23.2)$ \\
\hline Liver dysfunction & $126(29.2)$ \\
\hline
\end{tabular}

IQR: interquartile range; MRI: magnetic resonance imaging.

was numerically more prevalent in the PsA population than in the general population. Additionally, compared to patients without hyperlipidemia, patients with hyperlipidemia had higher BMI $(p=0.006)$ and higher rate of hyperuricemia $(\mathrm{p}=0.004)$, HTN $(\mathrm{p}=0.001)$, and liver dysfunction $(\mathrm{p}=$ $0.014)$. Interestingly, $89.7 \%$ of patients fulfilled CASPAR 
criteria for PsA, $63 \%$ fulfilled ASAS criteria for axial joint spondyloarthritis (SpA), and $98.2 \%$ fulfilled ASAS criteria for peripheral SpA. Table 2 shows types of treatments prescribed.

\section{DISCUSSION}

In this large-scale, multicenter study, we found a pooled PsA prevalence of $14.3 \%$ among patients with psoriasis (range, 8.8-20.4\%), similar to that in Western countries.

Psoriasis has been considered relatively rare among Asians, with a reported prevalence of $0.1 \%{ }^{2,3}$, although the rate is reported to be increasing ${ }^{14}$. Various reports have estimated the prevalence of PsA among patients with psoriasis in non-Asian populations to be $20-34 \%{ }^{7,15,16}$. In contrast, Fan, et al found the prevalence of PsA among a Chinese population to be only $5 \%$, while Yang, et al, reported a similar prevalence of $5.8 \%$; several other studies have reported rates of $\leq 10 \%{ }^{10,17}$. The prevalence of PsA among Japanese populations has been reported to be even lower, at $1 \%^{5}$. However, in keeping with our clinical experience that the prevalence of PsA among Japanese patients is actually higher than $1 \%$, we identified prevalence rates of up to $20.4 \%$ among Japanese patients with psoriasis.

The comparative prevalence rates found in our study and those reported in Western populations may be related to both environmental factors and improved diagnostic tools. First, the Westernization of the Japanese lifestyle has been associated with the increased prevalence of several risk factors for psoriasis, including family history of psoriasis, obesity, and hyperlipidemia. Additionally, the prevalence of several risk factors for PsA, including obesity, female sex, and presence of severe psoriasis, nail lesions, Koebner's phenomenon, and hyperlipidemia, have also increased ${ }^{18,19,20,21}$. In our study, a significant correlation was found between PsA and hyperlipidemia. Among the general Japanese population, hyperlipidemia is present in about $9.8 \%$ of adult men and $14.7 \%$ of adult women; both figures have been rising annually 22 . Thus, the rising rates of hyperlipidemia in Japan may contribute to the increase in the number of Japanese patients with PsA. Because the PsA prevalence rates differed among the institutions we surveyed, we investigated the relationships

Table 2. Treatment (current users) at 3 institutions.

\begin{tabular}{lc}
\hline Drug & $\mathrm{N}(\%)$ \\
\hline Nonsteroidal antiinflammatory drugs & $131(30.4)$ \\
Methotrexate & $174(40.4)$ \\
Salazosulfapyridine & $71(16.5)$ \\
Cyclosporine & $15(3.5)$ \\
Other disease-modifying antirheumatic drugs & $11(2.6)$ \\
Infliximab & $19(4.4)$ \\
Adalimumab & $65(15.1)$ \\
Etanercept & $6(1.4)$ \\
Ustekinumab & $10(2.3)$ \\
Steroids & $24(5.6)$ \\
\hline
\end{tabular}

of the above-mentioned risk factors with the patient populations, but did not find any significant correlations. Site-specific differences in PsA prevalence in our study may be secondary to differences in severity of psoriasis among regions. Prevalence of PsA among psoriasis is generally higher among patients with more severe psoriasis, which suggests the need for further investigation ${ }^{19}$.

A second possible reason for the increase in the prevalence of PsA is improved diagnostic methods for and the increased awareness of PsA. Previously, studies in Japanese patients were hindered by small datasets, due in part to poor recognition of the disease and absence of classification criteria prior to 2006. Moreover, diagnoses of PsA were typically made by dermatologists alone. We obtained more definitive PsA diagnoses by recruitment of rheumatologists to collaborate with dermatologists. To ensure appropriate diagnosis of PsA in the future, cooperation among specialists, as well as the introduction of objective PsA screening tools easily applied in daily practice, will be important. While our study did not include a formal screening tool, instruments have become available such as the Psoriatic Arthritis Screening and Evaluation Tool, which facilitates PsA diagnosis using a 1-page questionnaire ${ }^{23}$. The use of such tools will likely bring about more rapid identification of PsA and appropriate referral to a rheumatologist.

In past studies from Europe and North America, polyarthritis has been the most common distribution of arthritis, followed by asymmetrical oligoarthritis; relatively few cases demonstrated isolated distal interphalangeal space disease or arthritis mutilans ${ }^{10}$. The results of the current study corroborate previous findings. Moreover, the most common skin disease type found in this study was psoriasis vulgaris, similar to previous findings ${ }^{7,16}$. In addition, treatment choices in our study were consistent with the Group for Research and Assessment of Psoriasis and Psoriatic Arthritis treatment recommendations ${ }^{24}$.

Our study had several limitations. First, all PsA specialists did not necessarily use the same classification criteria in their respective clinical practices, thus introducing possible misclassificaton bias in the subject population. Haroon, et al, previously reported that up to $30 \%$ of patients not known to have PsA did indeed have PsA after a rheumatologic assessment ${ }^{25}$. Therefore our finding may still be an underestimate. Nonetheless, in our study, concordance with CASPAR and both ASAS peripheral and axial SpA classification criteria was $92.1 \%, 98.8 \%$, and $54.0 \%$, respectively. Only 1 of 431 cases failed to fulfill the former 2 criteria, indicating that there were no major errors in selecting the subject population. Second, multifactorial diagnostic limitations are possible, including inherent limitations in the cross-sectional study design. For example, as many patients experienced skin symptoms initially, subsequent treatment with antiinflammatory or immunosuppressant therapies could have modified clinical PsA symptoms. Imaging tests, which

Personal non-commercial use only. The Journal of Rheumatology Copyright @ 2015 . All rights reserved. 
may have clarified disease activity, were not performed on all patients. Third, patients seen in our tertiary care centers may have higher rates of PsA than patients seen in community dermatology clinics, a potential source of nondifferential bias that may affect the results of our study.

Our study warrants confirmation in other Asian populations to examine whether these prevalence numbers are applicable in other populations. While past epidemiological surveys have typically been single-institution, this study gathered data from 3 relatively large municipal hospitals. Hence, we believe our findings are adequately representative of the status of patients throughout Japan.

The maximum prevalence of PsA in patients with psoriasis in Japan was $20.4 \%$ in our study, similar to rates reported in Western countries. It is higher than previously reported prevalence rates in Asia. Clinical features were also similar to those observed in Western countries. Additional studies are required to determine whether these rates are also higher in other Asian countries.

\section{REFERENCES}

1. Taylor W, Gladman D, Helliwell P, Marchesoni A, Mease P, Mielants $\mathrm{H}$, et al. Classification criteria for psoriatic arthritis. Development of new criteria from a large international study. Arthritis Rheum 2006;54:2665-73.

2. Chang YT, Chen TJ, Liu PC, Chen YC, Chen YJ, Huang YL, et al. Epidemiological study of psoriasis in the national health insurance database in Taiwan. Acad Derm Venereol 2009;89:262-6.

3. Parisi R, Symmons DP, Griffiths CE, Ashcroft DM. Global epidemiology of psoriasis: a systematic review of incidence and prevalence. J Invest Dermatol 2013;133:377-85.

4. Langley RG, Krueger GG, Griffiths CE. Psoriasis: epidemiology, clinical features, and quality of life. Ann Rheum Dis 2005;64 Suppl 2:ii18-23.

5. Kawada A, Tezuka T, Nakamizo Y, Kimura H, Nakagawa H, Ohkido M, et al. A survey of psoriasis patients in Japan from 1982 to 2001. J Dermatol Sci 2003;31:59-64.

6. Ibrahim G, Waxman R, Helliwell PS. The prevalence of psoriatic arthritis in people with psoriasis. Arthritis Rheum 2009;61:1373-8.

7. Reich K, Kruger K, Mossner R, Augustin M. Epidemiology and clinical pattern of psoriatic arthritis in Germany: a prospective interdisciplinary epidemiological study of 1511 patients with plaque-type psoriasis. Br J Dermatol 2009;160:1040-7.

8. Gladman DD, Antoni C, Mease P, Clegg DO, Nash P. Psoriatic arthritis: epidemiology, clinical features, course, and outcome. Ann Rheum Dis 2005;64 Suppl 2:ii14-7.

9. Baek HJ, Yoo CD, Shin KC, Lee YJ, Kang SW, Lee EB, et al. Spondylitis is the most common pattern of psoriatic arthritis in Korea. Rheumatol Int 2000;19:89-94.
10. Fan X, Yang S, Sun LD, Liang YH, Gao M, Zhang KY, et al. Comparison of clinical features of HLA-Cw*0602-positive and -negative psoriasis patients in a Han Chinese population. Acta Derm Venereol 2007;87:335-40.

11. Takahashi H, Nakamura K, Kaneko F, Nakagawa H, lizuka H. Japanese Society for Psoriasis Research. Analysis of psoriasis patients registered with the Japanese Society for Psoriasis Research from 2002-2008. J Dermatol 2011;38:1125-9.

12. Rudwaleit M, van der Heijde D, Landewe R, Akkoc N, Brandt J, Chou CT, et al. The Assessment of SpondyloArthritis international Society classification criteria for peripheral spondyloarthritis and for spondyloarthritis in general. Ann Rheum Dis 2011;70:25-31.

13. Moll JM, Wright V. Psoriatic arthritis. Semin Arthritis Rheum 1973;3:55-78.

14. Kubota K, Sato T, Ooba N, Kamijima Y. Epidemiology using national database. The 28th Annual Meeting of the Japanese Society for Psoriasis Research 2013;Suppl:ii76.

15. Zachariae H. Prevalence of joint disease in patients with psoriasis: implications for therapy. Am J Clin Dermatol 2003;4:441-7.

16. Mease PJ, Gladman DD, Papp KA, Khraishi MM, Thaci D, Behrens $\mathrm{F}$, et al. Prevalence of rheumatologist-diagnosed psoriatic arthritis with psoriasis in European/North American dermatology clinics. J Am Acad Dermatol 2013;69:729-35.

17. Yang Q, Qu L, Tian H, Hu Y, Peng J, Yu X, et al. Prevalence and characteristics of psoriatic arthritis in Chinese patients with psoriasis. J Eur Acad Dermatol Venereol 2011;25:1409-14.

18. Armstrong AW, Harskamp CT, Dhillon JS, Armstrong EJ. Psoriasis and smoking: a systematic review and meta-analysis. Br J Dermatol 2014;170:304-14.

19. Soltani-Arabshahi R, Wong B, Feng BJ, Goldgar DE, Duffin KC, Krueger GG. Obesity in early adulthood as a risk factor for psoriatic arthritis. Arch Dermatol 2010;146:721-6.

20. Eder L, Shanmugarajah S, Thavaneswaran A, Chandran V, Rosen $\mathrm{CF}$, Cook RJ, et al. The association between smoking and the development of psoriatic arthritis among psoriasis patients. Ann Rheum Dis 2012;71:219-24

21. Wu S, Li WQ, Han J, Sun Q, Qureshi AA. Hypercholesterolemia and risk of incident psoriasis and psoriatic arthritis in US women. Arthritis Rheumatol 2014;66:304-10.

22. 2012 National Health and Nutrition Survey, Japan. Tokyo: Ministry of Health, Labor and Welfare; 2012.

23. Husni ME, Meyer KH, Cohen DS, Mody E, Qureshi AA. The PASE questionnaire: pilot-testing a psoriatic arthritis screening and evaluation tool. J Am Acad Dermatol 2007;57:581-7.

24. Ritchlin CT, Kavanaugh A, Gladman DD, Mease PJ, Helliwell P, Boehncke WH, et al. Treatment recommendations for psoriatic arthritis. Ann Rheum Dis 2009;68:1387-94

25. Haroon M, Kirby B, Fitzgerald O. High prevalence of psoriatic arthritis in patients with severe psoriasis with suboptimal performance of screening questionnaires. Ann Rheum Dis 2013;72:736-40. 